\title{
SPRACHWISSENSCHAFT
}

\author{
Joanna Szczęk*
}

\section{VON ABSAGEBRIEFEN ZU ABSAGEGENERATOREN \\ - KONSTANZ UND WANDEL IN FORM UND INHALT VON DEUTSCHEN ABSAGESCHREIBEN AUF BEWERBUNGEN}

Jede Änderung und jeder Wandel treibt den Fortschritt an. Es wird zwar oft angenommen, dass die Änderung eine neue und bessere Qualität einführen soll, aber erst die alltägliche Praxis kann das verifizieren. Dieselbe Regel findet auch im Bereich der schriftlichen Kommunikation Anwendung, da auch in dieser Sphäre der menschlichen Kommunikation ein beobachtbarer Wandel zu verzeichnen ist, der sich in Bezug auf die Form der zu beantwortenden Korrespondenz vollzogen hat, und v.a. den Möglichkeiten nach der Einführung unterschiedlicher Medien zu verdanken ist.

Diese Situation betrifft auch die Antworten auf Bewerbungen. Solche Schreiben sind nämlich als Frucht der Korrespondenzregel zu betrachten, dass es sich gehört, auf die eingehende Korrespondenz zu reagieren.

Die Antworten auf Bewerbungen können zusagenden oder ablehnenden Charakters sein. Im zweiten Falle werden die Antworten durch bestimmte Vorschriften geregelt, die im Weiteren besprochen werden, und weisen sowohl in Form als auch in den gebrauchten Formulierungen weitgehende Formelhaftigkeit auf (vgl. Szczęk 2015a, im Druck). Diese betrifft v.a. den Inhalt, den auch eine bestimmte Form und Struktur von Absagebriefen erzwingen. Und gerade die Absagebriefe scheinen im Laufe der Zeit einen gewissen Wandel vollzogen zu haben.

Diese Varianz in Bezug auf die Form und den Inhalt in den Absageschreiben nachzuweisen und Entwicklungstendenzen der Absageschreiben auf Bewerbungen

* Dr. habil. Joanna Szczęk, Universität Wrocław, Institut für Germanische Philologie, Lehrstuhl für Angewandte Linguistik, 50-140 Wrocław, Pl. Nankiera 15b. 
als Textsorten zu untersuchen und zu umreißen, ist das Ziel des vorliegenden Beitrags. Die Grundlage der Analyse bilden 253 authentische deutsche Absagebriefe, die im Zeitraum vom 2001 bis 2012 gesammelt wurden.

\section{Absageschreiben - Einige Bemerkungen zur Textsorte}

Absageschreiben, Absagebriefe oder Ablehnungsschreiben sind Bezeichnungen für alle Texte ablehnenden Charakters, in denen in Bezug auf die frühere Bitte, ein Angebot oder einen Vorschlag eine ablehnende Antwort erteilt wird. Zu dieser Textsorte wird in verschiedenen Kommunikationssituationen gegriffen, in denen eine klare Stellungnahme bzw. Entscheidung gefordert wird. Eine breite Palette der Situationen, in denen eine Absage formuliert werden kann, mag am Beispiel der Briefsteller und der Ratgeberliteratur veranschaulicht werden. So findet man z.B. Lubbers (1982) Beispiele für Absagen bei anderen Anlässen, die unter den ,persönlichen Briefen des Privatmanns“ zu finden sind. Es ist ein breiter Katalog von gesellschaftlichen Anlässen, im Falle deren eine Reaktion in Form einer Absage möglich wäre, z.B.: Beispiele für Absagen bei familiären Festen wie Hochzeitsfeier (S. 51), Silberhochzeit (S. 53), Weihnachten (S. 55) und bei anderen Gelegenheiten: Cocktailpartie (S. 56), Skatrunde (S. 57), Absage eines Kameraden (S. 58), Kaffeeklatsch (S. 59), Tee (S. 61), Gartenparty (S. 63), Forellenangeln (S. 64), Karnevalsparty (S. 65), Geselligkeit (S. 66), Segeltörn (S. 68), Abend am Kamin (S. 69), Dia-Schau (S. 70), Chinesen-Rallye-Party (S. 71). Hovermann (2009) dagegen formuliert einige Ratschläge, wie man negative Informationen freundlich und konstruktiv vermitteln soll, und liefert dazu 63 Musterbriefe und E-Mails für Absagen. Sie konzentriert sich auf das Geschäftsleben und betrachtet Absageschreiben als Schreiben, die Geschäftsbriefe in der Geschäftskorrespondenz sind. Absagen als Reaktionen auf bestimmte Stimuli können daher in folgenden Situation erfolgen: Absagen von vereinbarten Geschäftsterminen, Besprechungen, Konferenzen, Meetings, Vorträgen, Seminaren, Absagen bei festlichen Anlässen wie Empfang, Geburtstag, Betriebsjubiläum, Eröffnung eines Geschäfts, unternehmensinterne Absagen von Feiern: Weihnachtsfeier, Betriebsfest, Betriebsausflug, Absagen an Restaurants, Tagungsstätten, Hotels, Absagen auf Angebote, Absagen auf Anfragen, Absagen auf Bitten um eine Spende, Absagen auf Bewerbungen (ebd.).

In diesem Kontext sind Absageschreiben auf Bewerbungen Texte

die 1. auf der Grundlage einer schriftlichen Bewerbung ${ }^{1}$, 2. die von einem Kommunikationspartner (= Bewerber) 3. an den anderen Kommunikationspartner $(=$ den potentiellen Arbeitgeber) gerichtet wird, 4. von dem anderen Kommunikationspartner (= dem potentiellen

\footnotetext{
${ }^{1}$ Hier verstanden als Angebot.
} 
Von Absagebriefen zu Absagegeneratoren - Konstanz und Wandel in Form und Inhalt... 13

Arbeitgeber) verfasst werden, 5. und eine negative Entscheidung in Bezug auf die eingereichte Bewerbung enthalten. (Szczęk 2015, S. 48).

Im Korpus lassen sich drei Typen von Absageschreiben unterscheiden, die je nach der Intention des Senders ermittelt werden könne

- Aperitif-Briefe, deren Ziel ist es, über den Fortgang eines noch nicht beendeten Auswahlverfahrens zu informieren und dadurch vielleicht Appetit auf die künftige Stelle anzuregen,

- Eigentliche Absageschreiben, in denen eine klare Ablehnung/Absage in Bezug auf die in der Bewerbung formulierte Bitte um Einstellung formuliert wird,

- Eisschreiben, in denen Ablehnung und Information über die Speicherung der Daten zur Bekundung des Interesses an einer künftigen Zusammenarbeit mitgeteilt wird.

Alle ermittelten Typen von Absagebriefen können als Varianten der Textsorte Absageschreiben betrachtet werden. Ihre hierarchische Textsortenzugehörigkeit kann in Anlehnung an das Modell von Heinemann/Heinemann (2002) folgendermaßen dargestellt werden:

Texttyp

Textsortenklasse 2

Textsortenklasse 1

Textsorte

Textsortenvariante:

Schema 1. Textsortenzuordnung der Absageschreiben in Anlehnung an Heinemann/Heinemann (2002) (vgl. Szczęk 2015, S. 161)

Dabei werden Textsortenvarianten als „Unterarten von Textsorten, die sich aus der Variation eines einmal produktiven Textmusters ergeben" definiert (Gansel/ Jürgens 2007, S. 72). Sie sind halboffiziellen Charakters und scheinen aus dem Bedürfnis heraus entstanden zu sein, dem Bewerber eine Antwort erteilen zu müssen. Im Kontext der umrissenen Kommunikationssituation kann man nämlich feststellen, dass die Antworten auf Bewerbungen auf einer Achse zwischen einer positiven Antwort (= Zusage) und einer negativen Antwort (= Absage) zu verorten sind, wobei die zwei grundlegenden Typen von Antworten: Zusage und Absage zwei Gegenpole auf dieser Reaktionen-Achse bilden, auf der dazwischen andere nicht so direkte Reaktionen oder Antworten zu platzieren sind. Man könnte es schematisch wie folgt darstellen: 


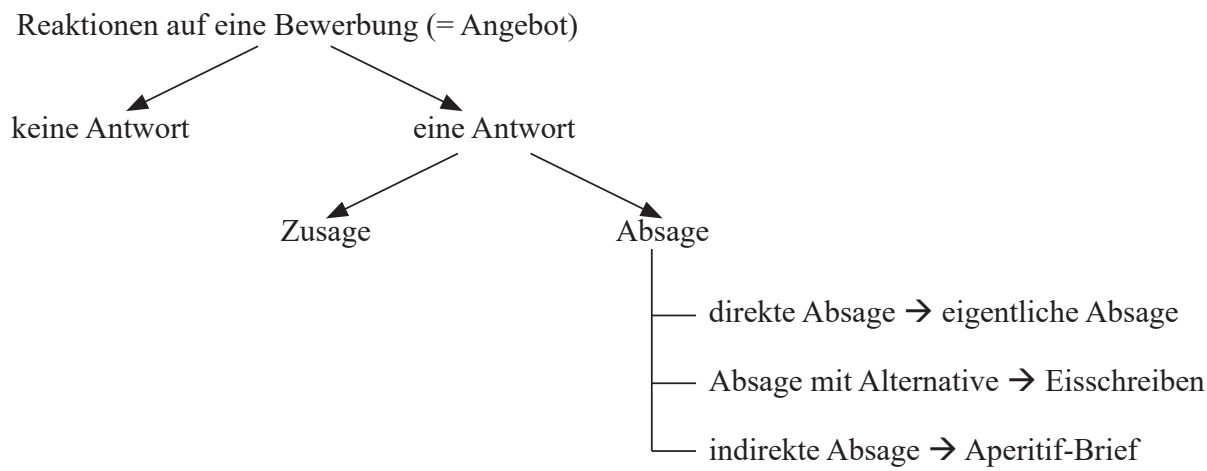

Schema 2. Typen von Reaktionen auf Bewerbungen

Im Grunde genommen handelt es sich hier um Strategien der Ablehnung, die nach Grein (2007, S. 407) folgende Sprechaktkombinationen im Deutschen umfassen können: Entschuldigung + Ablehnung + Begründung + Alternative, Entschuldigung + Ablehnung, Ablehnung + Alternative, Alternative, Entschuldigung + Ablehnung + Begründung, Ablehnung + Begründung + Alternative, Begründung + Alternative, Entschuldigung + Begründung. Bei Nixdrof (2002, S. 48) sind es: Nein, Will nicht, Erklärung, Bedauern, Alternative, Angriff, als ob, Vermeidung (Verschiebung, Themawechsel, Wiederholung, Witz). In den Absageschreiben sind es folgende Kombinationen, die in Bezug auf die mitgeteilte Absage unterschiedlich platziert werden können: nach dem ABSAGEN/ABLEHNEN: BEGRÜNDEN, BEDAUERN, TIPP, DANKEN, AUFFORDERN, und vor dem ABSAGEN/ABLEHNEN: BEDAUERN, BESTÄTIGEN, DANK, TIPP, BEGRÜNDEN, BITTE.

Man kann auch die These wagen, dass im Umgang mit Bewerbungen die eigentlichen Absageschreiben als primäre Textsorten zu betrachten sind, und erst im Laufe der Zeit die anderen zwei Textsortenvarianten entstanden sind, um einerseits den Anforderungen des Arbeitsmarktes und den Vorschriften des Gesetztes gerecht zu werden, und andererseits die Bedürfnisse der Bewerber zu befriedigen. Es ist in diesem Falle jedoch schwer, die goldene Mitte zu finden, wie es Müller/ Moser (2006) hervorheben, indem sie die Merkmale der Absageschreiben darstellen, die wichtig beim Erstellen dieser Bescheide sind. Aus der Perspektive des Empfängers (= Bewerbers) handelt es sich um folgende Aspekte: „Informationen zum Auswahlverfahren“, die als „Möglichkeit“ angesehen werden ,abgelehnte Bewerber positiv zu beeinflussen" (ebd., S. 347). Hinzu kommt auch das Timing der Absagen, bei dem zwei Möglichkeiten besprochen werden:

Einerseits gibt es gute Gründe, den Bewerber möglichst lange im Auswahlprozess zu belassen und ihm erst nach intensiver Informationssammlung eine Absage mitzuteilen. Auf diese Weise kann man dem Kandidaten, etwas Gutes' tun und ihm die besondere Wertschätzung seitens des Unternehmens mitteilen (ebd.). 
Andererseits aber wird davor gewarnt: „Allerdings könnten Bewerber eine verzögerte Reaktion auch als unorganisierte Verwaltung oder Schikane des Unternehmens interpretieren und eine Ablehnung besonders negativ erleben, wenn bereits umfassend Informationen gesammelt wurden“" (ebd.). Die zweite Möglichkeit sei eine frühzeitige Zustellung der Absage, die wiederum als Zurückweisung empfunden werden kann, denn ,eine schnelle und völlig unbegründete Absage könnte beim Bewerber allerdings auch zum Eindruck führen, dass sich das Unternehmen überhaupt nicht mit der Bewerbung beschäftigt hat" (ebd.). Deswegen wird in dem Kontext empfohlen, „das Resultat eines Vorstellungsinterviews ca. eine Woche intern ,zu bearbeiten' oder , sorgfältig abzuwägen', bevor eine Absage verschickt wird" (ebd.).

\section{Einige Bemerkungen zur Kommunikationsstruktur im Bewerbungsverfahren}

Die Kommunikation zwischen den Interaktanten im Bewerbungsprozess ist als eine sprachliche Handlung anzusehen, wobei sie ,grundlegend kooperativ zwischen einem Sprecher S und einem Hörer H erfolgt" (Redder 2010, S. 11).

In den Absageschreiben kommt es zur Interaktion zwischen Absageschreiber und Bewerber, die in eine bestimmte Gruppe und Gesellschaft eingebettet sind und sich in einem Interaktionsprozess befinden (vgl. Große 1976, S. 11). Die Interaktion verläuft nach bestimmten Regeln und sie als Mitglieder von bestimmten Gruppen übernehmen verschiedene Rollen, ,die sich innerhalb eines gegebenen Feldes sozialer Beziehungen aus dem wechselseitigen Bezug zwischen einer sozialen Position (...) und anderen, z.B. unter- oder gleichgeordneten Positionen (...) ergibt“ (Große 1976, S. 13). Hervorzuheben ist im Kontext der Absageschreiben der sequentielle Rollenwechsel von Sender und Empfänger, der von der kommunikativen Situation erzwungen wird, wie es Drescher (1994, S. 120) expliziert, ,es kommt seitens (...) des potentiellen Arbeitgebers in der Regel zu einer, sich aus der Arbeitsteilung ergebenden Aufspaltung der Sender- bzw. Empfängerrolle - etwa in Absender (= Unternehmen, Schreiber $=$ Sekretariat) und Verfasser (= Sachbearbeiter/in)“. Dabei werden in den beiden Sequenzen den Interaktanten bestimmte „Aufgaben“ zugeordnet, die man folgendermaßen darstellen kann: 


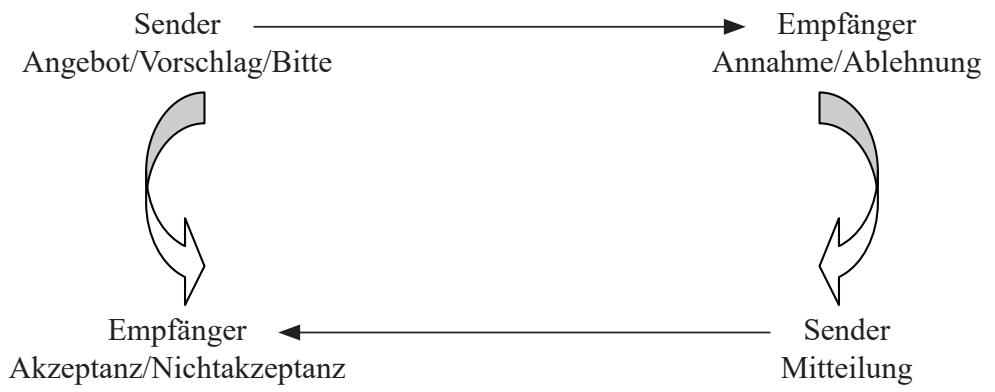

Schema 3. Rollenwechsel in der Sequenz Angebot/Vorschlag/Bitte - Annahme/Ablehnung (vgl. Szczęk 2015, S. 47)

Die beiden Interaktanten stehen in einem asymmetrischen Verhältnis zueinander, wobei dem Sender eine offizielle und dem Empfänger eine nichtoffizielle Rolle zukommt. Auf Grund dessen kann der Geltungsbereich der Absageschreiben als halboffiziell bestimmt werden, wie es am folgenden Schema gezeigt wird:

Kriterium:

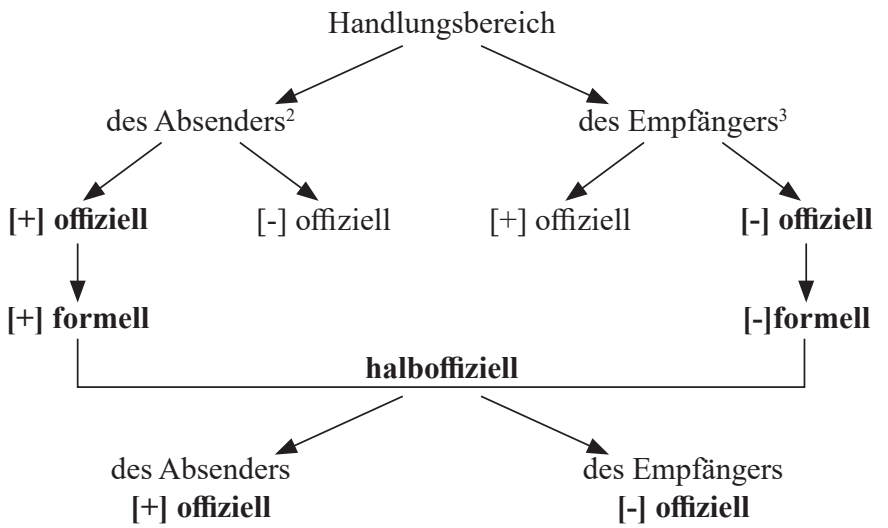

Schema 4. Darstellung des Handlungsbereichs in den Absageschreiben in Anlehnung an Ermert (1979, S. 76f.), eigene Darstellung

\section{Zum Umgang mit Bewerbungen - Bemerkungen zu Entwicklungstendenzen der Textsorte Absageschreiben}

Der im vorliegenden Beitrag angenommener Zeitraum, aus dem die Absageschreiben stammen, beträgt 12 Jahre. In Bezug auf die möglich beobachtbaren Entwicklungstendenzen mag es vielleicht nicht lang sein. Es lässt sich aber auf drei

\footnotetext{
$2^{2}=$ Absageschreiber.

${ }^{3}=$ Bewerber.
} 
wichtige Faktoren hinweisen, die sowohl den Inhalt als auch die Form von diesen Schreiben wesentlich beeinflusst und die Entwicklung der Textsorte bewirkt haben. Es handelt sich zum einen um Regelungen, die den Absageschreibern vom Gesetzgeber auferlegt werden. Zum anderen ist es die reiche Ratgeber-Literatur, die bestimmte Lösungen und Vorschläge für den Umgang mit Absageschreiben unterbreitet. Man kann seit einiger Zeit nämlich eine hohe verlegerische Produktivität in diesem Bereich anmerken.

Und zuletzt ist auch die Entwicklung der Medien nicht zu übersehen, die gewisse Änderungen eingeführt und Erleichterungen für Absageschreiber geschaffen hat, die am Beispiel der Absage-Generatoren sichtbar sind.

\subsection{Das Höchstalter ist bei den Bewerbern auf 30 Jahre beschränkt - Rechtliche Grundlagen für den Umgang mit Bewerbungen ${ }^{4}$}

Der korrekte und notwendige Umgang mit Bewerbungen wurde und wird durch eine gewisse Situation auf dem Arbeitsmarkt erzwungen. Es handelt sich v.a. um die Pflichten der Arbeitslosen gegenüber der „Agentur für Arbeit“, im Rahmen deren sie verpflichtet sind, die Beweise ihrer Bemühungen um Arbeitsstellen zu sammeln, um sie bei Bedarf als Beweise vorzeigen zu können. Diese Situation hatte zur Folge, dass auch die potenziellen Arbeitgeber "gezwungen“" wurden, jede eingehende Bewerbung entsprechend zu behandeln, d.h. auf jede Bewerbung eine Antwort zu geben. Dies erweist sich jedoch als eine schwer zu bewältigende Aufgabe, wie man in der Ratgeberliteratur liest:

Es ist sicher nicht angenehm, einem Bewerber, der sich Mühe mit der Zusammenstellung seiner Unterlagen gemacht hat, eine Absage zu erteilen. Manche Unternehmen drücken sich deshalb um diese unangenehme Aufgabe. Sie antworten entweder gar nicht oder schicken allen Bewerbern das gleiche fotokopierte Schreiben (Duden 1997, S. 236).

Im Laufe der Zeit ist das Verfassen von Absageschreiben zu einer massenweisen „Produktion“ geworden, was einen engen Zusammenhang zwischen der Wirklichkeit und der künstlich geschaffenen Situation entstehen lässt, dessen Frucht - die Absageschreiben - textlinguistischer Natur ist. Durch dieses häufige Verfassen von Absagen, die inzwischen zu einem Sammelobjekt geworden sind, sind jedoch die Glaubwürdigkeit und das Prestige dieser Textsorte wesentlich gesunken.

Erst seit 2006 wird der Umgang mit Bewerbungen in Deutschland durch das „Allgemeine Gleichbehandlungsgesetz“5 (= AGG) geregelt, nach dem die Absageschreiber sehr behutsam mit den eingegangenen Unterlagen umgehen sollen. Es

\footnotetext{
${ }^{4}$ Vgl. Szczęk (2015).

${ }^{5}$ Vgl. http://www.gesetze-im-internet.de/agg/BJNR189710006.html, Zugriff am 17.07.2012.
} 
gilt nämlich, „den Bewerbern diskriminierungsfrei zu antworten“6 und den Inhalt des Absageschreibens entsprechend dem AGG zu formulieren. Daraus ergibt sich, dass ,die im AGG vorgesehene Beweislastverteilung klar zu Lasten des Arbeitgebers geht" und man sich „bei der Absage möglichst vorsichtig und zurückhaltend ausdrücken" soll. Ein Absageschreiben darf vor allem keinen Hinweis auf eines der acht in $\S 1$ AGG genannten Kriterien (Rasse, ethnische Herkunft, Geschlecht, Religion oder Weltanschauung, Behinderung, Alter oder sexuelle Identität) enthalten ${ }^{7}$. Das Gesetz betrifft auch Auszubildende und Bewerber für ein Beschäftigungsverhältnis und ist für Arbeitgeber bindend. Es gilt zugleich als eine Art Orientierungshilfe für Absageschreiber, ,um Verstöße gegen das Benachteiligungsverbot zu vermeiden“ (Hovermann 2009, S. 79).

Aus diesem Grunde wäre z.B. eine solche Formulierung aus dem Absageschreiben dem Jahre 2002: Das Höchstalter bei den Bewerbern/innen ist auf 30 Jahre beschränkt heutzutage nicht zulässig. Bis auf dieses eine Beispiel weisen die das Korpus bildenden Absageschreiben keine weiteren Abweichungen und Verstöße gegen das Gesetz auf.

\subsection{Machen Sie sich die Mühe, eine Absage zu schreiben, die dem Bewerber einen Grund für die Absage nennt! - Zum Umgang mit Bewerbungen im Lichte der Ratgeber-Literatur}

Zur offiziellen Schriftkultur gehört immer noch, auf Anträge, Bewerbungen, Angebote u.Ä. eine schriftliche Antwort zu erteilen. Das betrifft auch Bewerbungen, die als Angebote seitens der Bewerber betrachtet werden können (vgl. Szczęk 2015), und wirkt sich auch auf das Zusammenstellen von Absagebriefen aus.

Das Umsetzen der Bestimmungen von AGG in die Tat hat sich auch auf die sog. Ratgeberliteratur ausgewirkt. Die Autoren der guten Ratschläge fürs Verfassen von Absageschreiben scheinen dabei in zwei Richtungen zu gehen, die als Kompromiss zwischen den Vorschriften von AGG und den Anforderungen des Arbeitsmarktes anzusehen sind. Es wird nämlich einerseits empfohlen, jeder Absage eine Begründung beizufügen, wofür man Beispiele unter Absagebriefen älteren Datums finden kann. In den neueren Absagen wird dagegen davon abgeraten, wie z.B. bei Hovermann (2009, S. 79): „Vermeiden Sie daher möglichst eine Rechtfertigung Ihrer Entscheidung. Die Begründung für eine Ablehnung könnte als Diskriminierung angesehen werden“".

Man kann sich die Frage stellen, wo die sog. goldene Mitte liegt? Diese kann man z.B. in den Ratschlägen in Bezug auf alle drei Typen von Absageschreiben

${ }^{6} \mathrm{Vgl.http}: / /$ www.azubee.de/fileadmin/user_upload/.pdf/azubee_allgemein/Bewerbern_diskriminierungsfrei_absagen.pdf, Zugriff am 24.07.2013.

${ }^{7}$ Vgl.http://www.azubee.de/fileadmin/user_upload/.pdf/azubee_allgemein/Bewerbern_diskriminierungsfrei_absagen.pdf, Zugriff am 23.07.2013. 
finden, wie es der folgenden Zusammenstellung zu entnehmen ist (vgl. Brucker 2008, S. 38f.):

- Aperitif-Briefe:

- Verabschieden Sie sich vom Telegrammstil der Massenschreiben mit dem Titel „Eingangsbestätigung“ und den Worten „Ihre Bewerbung ist angekommen. Wir werden uns zu gegebener Zeit bei Ihnen melden“.

- Nutzen Sie das Image des Mittelständlers, der sich gerade durch die persönliche Ansprache der Mitarbeiter von Großkonzernen abhebt.

- Machen Sie dem Bewerber von Anfang an klar, dass er bei Ihnen ein Individuum ist und nicht die Nummer 102 unter den Bewerbern. Der potenzielle Mitarbeiter soll schließlich Appetit auf Ihr Unternehmen bekommen.

- Eisschreiben:

- Betreiben Sie ganzheitliches Personalmarketing. Dazu gehört, professionell auf Initiativbewerbungen zu reagieren. Besonders, wenn es sich um gute Kandidaten handelt, zu denen Sie weiterhin Kontakt halten möchten.

- Formulieren Sie so, dass der Bewerber nicht das Gefühl hat, eine verkappte Absage zu erhalten.

- Senden Sie das eindeutige Signal aus, dass er prinzipiell zum Unternehmen passt. Die Kernbotschaft lautet: „Wir brauchen Leute wie Sie, wenn auch nicht gerade in diesem Moment. Deshalb ist uns der weitere Kontakt zu Ihnen wichtig".

- Eigentliche Absageschreiben:

- Man sieht sich immer zweimal im Leben, heißt ein Sprichwort. Mit Blick auf den Fachkräftemangel kann es sich kein Unternehmen mehr erlauben, Leute auf ewig zu vergraulen. Vielleicht möchten Sie den Bewerber, wenn er über bessere Qualifikationen verfügt, zu einem späteren Zeitpunkt als Mitarbeiter gewinnen. Denken Sie daran: Mit jedem nach außen gerichteten Schreiben präsentieren Sie sich als Arbeitgeber. Es spricht sich herum, wie eine Firma mit Bewerbern umgeht.

Die Verfasser (= Absageschreiber) verwenden dabei bestimmte Muster, in denen eine gewisse Formelhaftigkeit nicht nur in Form, sondern auch in Sprache erkennbar ist, was einerseits die sog. ,Absagesammler“8 und andererseits Textforscher bestätigen":

(...) negative Antworten auf Bewerbungen stellen eine in Verwaltungen wie Unternehmen häufig anfallende kommunikative Aufgabe dar. Stehen für die Bewältigung dieser Aufgabe

${ }^{8}$ Gemeint sind Bewerber, die Beweise ihrer ständigen Bemühungen um Arbeit sammeln müssen, um sie bei den zuständigen Behörden vorweisen zu können.

${ }^{9}$ Als Beweis dafür kann man fertige Schablonen für Absageschreiben, sowie Absagegeneratoren anführen, im Falle derer man nur die gewählte Formulierung anzuklicken braucht, vgl. z.B. http:// www.absage-bewerbung.de/absagen-generator-absagen-individuell-zusammenstellen/, Zugriff am 13.09.2013. 
standardisierte Briefe zur Verfügung, so liegt die Vermutung nahe, dass bei deren Anfertigung auch sprachliche Muster Verwendung finden (Drescher 1994, S. 117).

Die Autoren der Ratgeber für Erstellen der Korrespondenz empfehlen auch, gewisse Strategien und Formulierungen beim Verfassen von Absagen zu verwenden. Im Duden (1997, 2005a, S. 239ff. und 2005b, S. 129ff.) werden drei Möglichkeiten vorgeschlagen, mit Absagen geschickt umzugehen. Sie enthalten folgende thematisch-strukturelle Bausteine:

1. Möglichkeit 1:

- Dank für die ausführliche/übersichtliche/ansprechende Bewerbung aussprechen

- Hinweis auf die vielen Bewerbungen, die auf die Stellenanzeige eingegangen sind, geben

- Grund für die lange Bearbeitungszeit nennen: Auswahl war wegen der gleichen Qualifikation der Bewerber schwierig

- Dem Bewerber mitteilen, dass er zwar in die engere Wahl gekommen ist, aber die Wahl schließlich auf einen anderen fiel

- Unterlagen mit diesem Brief zurück

- Ermutigung: sicher bei der nächsten Bewerbung Erfolg

2. Möglichkeit 2:

- Dank für die ausführliche/übersichtliche/ansprechende Bewerbung aussprechen

- Dem Bewerber mitteilen, dass er den Anforderungen der ausgeschriebenen Stelle voll entsprach, aber seine Gehaltsvorstellungen über den Etat für diese Stelle hinausgehen

- Mut machen: Bestimmt wird eine Bewerbung bei einem größeren Unternehmen erfolgreich sein

- Unterlagen mit diesem Brief zurück

3. Möglichkeit 3:

- Dank für die ausführliche/übersichtliche/ansprechende Bewerbung aussprechen

- Dem Bewerber erklären, dass es mehrere Interessenten mit hoher Qualifikation gab, einer von ihnen jedoch bereits über einschlägige Erfahrungen im ausgeschriebenen Arbeitsbereich verfügt

- Wertschätzung zeigen: Sie hätten den Bewerber gerne kennen gelernt, aber die Stelle kann leider nur einmal besetzt werden

- Um Verständnis bitten

- Unterlagen mit diesem Brief zurück

- Erfolg wünschen

Man findet auch eine Reihe von Ratschlägen, die sich auf die thematische Struktur der Absagen beziehen (vgl. Duden 2010, S. 234), z.B.: „Danken Sie für die Bewerbung oder das Angebot“; ,Sagen Sie etwas Positives über den Bewerber 
oder Anbieter und bedauern Sie die Ablehnung“; „Erklären Sie, warum Sie einen anderen bevorzugt haben“; „Falls denkbar: Schreiben Sie, dass Sie den Bewerber gern beim nächsten Mal wieder berücksichtigen möchten“; „Wünschen Sie dem Bewerber für die Zukunft alles Gute“.

Ergänzend kann man noch fertige Muster von Absageschreiben nennen, die einem online zur Verfügung stehen und an die jeweilige Situation beliebig angepasst werden können, indem entsprechende Daten ergänzt werden, wie z.B. Muster 2 der Absage zu Bewerbung ${ }^{10}$ :
Max Mustermann
Musterstraße 1
11111 Musterstadt
Musterstadt, den 01.01.2013
Sehr geehrte Herr/ Frau Mustermann, schaft ein Teil unseres Teams zu werden, möchten wir uns herzlich bedanken. bekommen haben. Das Auswahlverfahren war durchaus nicht leicht. einen anderen Bewerber entschieden haben.
Ihre Bewerbungsmappe fügen wir dem Schreiben hinzu.
Wir wünschen Ihnen für Ihren weiteren beruflichen Erfolg alles Gute.
Mit freundlichen Grüßen
XXX

Sie haben sich bei uns auf eine Ausbildungsstelle/ Arbeitsstelle beworben. Für Ihre Bereit-

Wir hoffen Sie können sich vorstellen, dass wir zahlreiche Angebote von guten Bewerbern

Dennoch tut es uns leid, Ihnen keine bessere Nachricht übermitteln zu können, da wir uns für

Das oberste zu beachtende Prinzip lautet aber: „Versetzen Sie sich in die Lage des Empfängers!“ (Duden 2010, S. 234), von dem weitere Ratschläge folgender Art abgeleitet werden: „Geben Sie dem abgelehnten Bewerber das Gefühl, individuell wahrgenommen worden zu sein. Versuchen Sie, ihm auch irgendeine positive Bewertung zu geben - natürlich sollte diese nicht unrealistisch sein.“ (ebd.). Zusätzlich findet man Hinweise zur Verfassung und Formulierung der Absagen, z.B.: „Danken Sie für die Bewerbung oder das Angebot. Sagen Sie etwas Positives über den Bewerber (...) und bedauern Sie die Ablehnung. Erklären Sie, warum Sie einen anderen bevorzugt haben. (...) Schreiben Sie, dass Sie den Bewerber beim nächsten Mal wieder berücksichtigen möchten. Wünschen Sie dem Bewerber für die Zukunft alles Gute“ (ebd.). Dabei hebt man auch die Funktion der Absageschreiben bei der Aufrechterhaltung des Kontakts und den

${ }^{10} \mathrm{Vgl}$. http://www.arbeitstipps.de/absage-zur-bewerbung-formulieren-muster-und-vorlagen. html\#.VgE9hZdBFnA, Zugriff am 22.09.2015. 
Nutzen für die jeweilige Firma hervor, z.B.: „Lieblose Absagen auf Bewerbungen schaffen immer auch ein negatives Image für Ihr Unternehmen“ (ebd.) und dies sollte eigentlich nicht passieren, ,denn ein gut formuliertes Absageschreiben (...) kann noch viel mehr: den Bewerber bei der Stange halten, die Firma, positionieren, Werbung machen, sich als guter Arbeitgeber präsentieren“ (Markt \& Technik 2005, S. 64).

\subsection{Stellen Sie die Bewerbungsabsagen individuell zusammen! - Zu den Absagen-Generatoren online}

Ein Novum im Umgang mit den Bewerbungen stellen Absagen-Generatoren dar, die online zugänglich sind und es möglich machen, die Absagebriefe ,ganz individuell“" zu gestalten. Man hat dabei unter einigen Vorschlägen für die einzelnen Bausteine des Textes zu wählen, die man nur anzuklicken braucht. Die Palette an Wahlmöglichkeiten scheint dabei nicht besonders groß zu sein, ${ }^{11}$ z.B.:
Anrede
- Sehr geehrte Frau [Empfänger],
Sehr geehrter Herr [Empfänger],
Einleitung
- vielen Dank für Ihre Bewerbung.
vielen Dank für Ihre Bewerbung und Ihr Interesse an einer Anstellung in unserem Unternehmen.
wir bedanken uns für Ihre Bewerbung und das damit zum Ausdruck gebrachte Interesse an einer Mitarbeit in unserem Unternehmen.
vielen Dank für Ihr Schreiben und die Unterlagen über Ihren bisherigen Werdegang.
wir bedanken uns für Ihre Bewerbung und die Zusendung Ihrer Bewerbungsunterlagen.
wir bedanken uns für die Einreichung Ihrer Bewerbungsunterlagen und Ihrem Interesse an einer Mitarbeit in unserem Unternehmen.

\section{Hauptteil}

- Wir müssen Ihnen leider mitteilen, dass wir Sie bei der Besetzung der Stelle nicht berücksichtigen können. Wir bedauern, Ihnen keinen günstigeren Bescheid geben zu können.

Leider müssen wir Ihnen mitteilen, dass wir Ihnen derzeit keine Ihren Vorstellungen und Kenntnissen entsprechende Position in unserem Unternehmen anbieten können. Wir bedauern, Ihnen keine positive Rückmeldung geben zu können.

Wir haben inzwischen unsere Vorauswahl getroffen. Leider konnten wir Ihre Bewerbung nicht in die engere Wahl ziehen - wir haben uns für einige Kandidatinnen und Kandidaten entschieden, deren Profil noch genauer den Anforderungen der ausgeschriebenen Position entspricht.

Ihre Eigeninitiative und Ihr Interesse an einer Tätigkeit in unserem Unternehmen haben

${ }^{11} \mathrm{Vgl}$. http://www.absage-bewerbung.de/absagen-generator-absagen-individuell-zusammenstellen/, Zugriff am 22.09.2015, siehe auch http://www.bewerbungsplan.de/absageschreiben-fuerdie-bewerbung/, Zugriff am 22.09.2015, http://www.business-solution-tools.de/business-solutiontools.de/tools/online-generatoren/absageschreiben-bewerber/, Zugriff am 22.09.2015. 
uns gefallen. Leider können wir Ihnen zur Zeit keine passende Position anbieten. Ihre Bewerbungsunterlagen erhalten Sie daher mit Dank zurück.

Wir haben Ihre Bewerbung im Kollegenkreis eingehend besprochen. Im Ergebnis müssen wir jedoch leider mitteilen, dass wir Ihnen die gewünschte Position nicht anbieten können. Wir bedauern, Ihnen keine günstigere Nachricht geben zu können und reichen die uns überlassenen Bewerbungsunterlagen anbei zurück.

Es ist uns nicht leicht gefallen, unter der Vielzahl qualifizierter Bewerbungen eine Auswahl zu treffen. Leider können wir im engeren Kreis der Bewerber nur diejenigen Kandidaten berücksichtigen, deren Qualifikationsprofil den spezifischen Anforderungen der ausgeschriebenen Stelle entspricht.

\section{Danksagung}

- Wir bedanken uns für das unserer Sozietät entgegengebrachte Interesse und wünschen Ihnen weiterhin viel Erfolg und alles erdenklich Gute.

Wir danken Ihnen für Ihr Interesse an einer Mitarbeit in unserem Unternehmen und wünschen Ihnen für Ihren weiteren beruflichen Weg alles Gute und viel Erfolg.

Wir bedauern sehr, Ihnen keine günstigere Nachricht geben zu können, und bedanken uns für das unserem Hause entgegengebrachte Interesse.

Wir danken Ihnen für Ihre Mühe, die Sie sich mit der Bewerbung gemacht haben und wünschen Ihnen alles Gute für Ihren weiteren Weg.

Bitte sehen Sie unsere Entscheidung nicht als Bewertung Ihrer Kenntnisse und Fähigkeiten an. Für Ihren weiteren Berufsweg wünschen wir Ihnen viel Erfolg sowie alles Gute für Ihre persönliche Zukunft.

\section{Grußformel}

- Mit freundlichen Grüßen

Mit nochmaligem Dank und freundlichen Grüßen

Dabei fällt sofort auf, dass man es hier mit keinem individuellen Stil zu tun hat. Es sind eher Wendungen floskelhaften Charakters, die von allen benutzt werden. Dies solle nach Meinung der Experten aus zwei Gründen erfolgen. Zum einen ist es die Menge:

Für eine Stellenanzeige gehen mitunter mehrere hundert Bewerbungen ein. Allein schon wegen dieser Menge ist es in der Praxis schlicht unmöglich, jede einzelne Bewerbung mit einem individuellen, eigens dafür formulierten Schreiben zu beantworten. Zudem würden sich die Formulierungen zwangsläufig wiederholen, denn die Möglichkeiten, eine Absage auszudrücken, sind begrenzt ${ }^{\star 12}$.

Zum anderen sind es die Bestimmungen des AGG: „Alle Bewerber müssen gleich behandelt und keiner darf diskriminiert werden. Eine Konsequenz daraus ist, dass Unternehmen gut beraten sind, eine Absage nicht näher zu begründen. (...) Insofern ist es dem Gesetz geschuldet, dass die meisten Bewerber nicht (mehr) erfahren, warum oder woran ihre Bewerbung gescheitert ist"“ (ebd.).

${ }^{12}$ Vgl. http://www.bewerbungsplan.de/absageschreiben-fuer-die-bewerbung/, Zugriff am 22.09.2015. 


\subsection{Viel Erfolg für Ihre berufliche Zukunft und richten Sie sich doch einfach mit uns ein! - Zu Werbeelementen in den Absageschreiben}

In den Absageschreiben findet man auch Textpassagen, die einen Werbecharakter aufweisen, obwohl ein Absageschreiben als Werbung eher schwer vorstellbar ist. Die Werbung wird meistens nach der Bestätigungs- und Dankesformel platziert, z.B.: Das Theater am Halleschen Ufer, 1993 als zentrale Spielstätte der freien Gruppen Berlins eingerichtet, ist heute einer der profiliertesten und anerkanntesten Spiel- und Produktionsorte für die Darstellenden Künste mit Schwerpunkt im Tanz-, Performancebereich. Man findet auch direkte Werbung, die nach der Absage ausgedrückt wird, z.B. in dem Absageschreiben von IKEA: Bis dahin wünschen wir Ihnen viel Erfolg für Ihre berufliche Zukunft und richten Sie sich doch einfach mit uns ein!, wobei durch die Anwendung des Imperativsatzes versucht wird, die Aufmerksamkeit des Empfängers/Lesers von der in diesem Schreiben mitgeteilten Absage abzulenken. Dieselbe Vorgehensweise finden wir auch in der Absage von Yello Strom: Sie denken, dass Sie gut zu Yello passen - auch wir sind dieser Meinung. Jedoch sind die Mitarbeiter genauso zufrieden wie unsere Kunden: daher gibt es zur Zeit leider keine Position im Marketing.

Der Höhepunkt sind Absageschreiben, die als Ganzes der Werbung des jeweiligen Unternehmens dienen und in der Stilistik der jeweiligen Firma formuliert werden, die dem Warensortiment der Firma entspricht, wobei die Absageschreiber scheinen, die Grenzen ganz überschritten zu haben, z.B.:

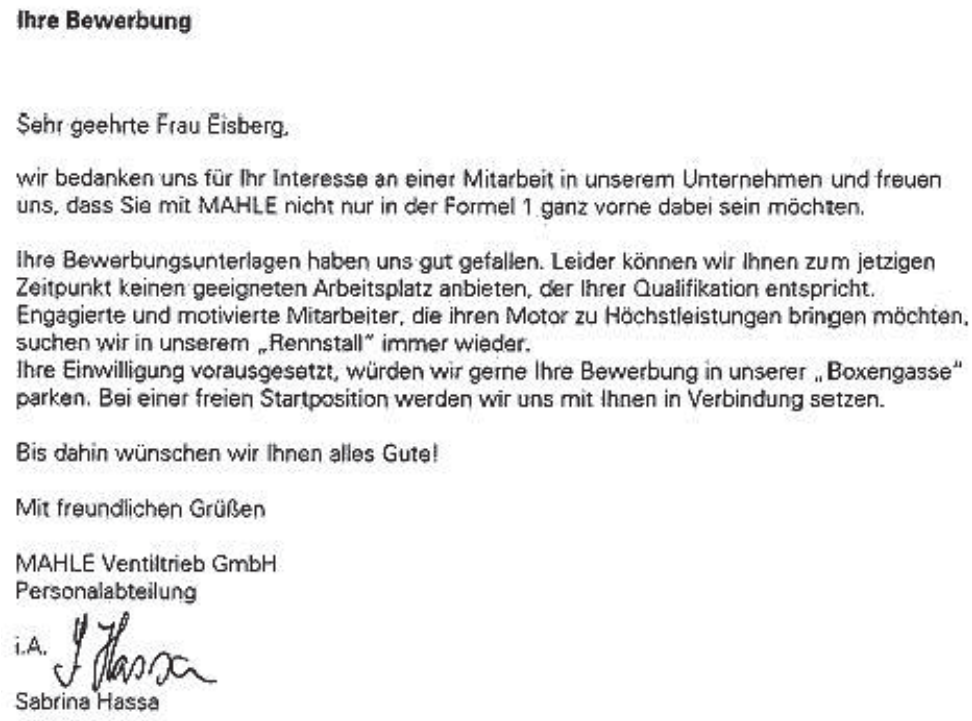

Abbildung 1. Beispiel für eine Absage mit Werbeinhalt Vgl. www.spiegel.de/unispiegel/jobundberuf/html, Zugriff am 8.07.2014 
Von Absagebriefen zu Absagegeneratoren - Konstanz und Wandel in Form und Inhalt... 25

\subsection{Exkurs: Um ein Haar hätte ich mich bei Ihnen beworben ${ }^{13}$ - Zum humoristischen Umgang mit Bewerbungen}

Im Kontext der Fragen nach dem Umgang mit Bewerbungen ist noch auf eine Erscheinung zu verweisen, die von einer gewissen Distanz der Bewerber zeugt und im krassen Gegensatz zu den Bemühungen der Bewerbungstrainer steht. Es handelt sich nämlich um den humoristischen Umgang mit Absageschrieben, die als eine Strategie gedeutet werden können, mit dieser Problematik fertig zu werden.

In diesem Kontext sind Wettbewerbe zu erwähnen, in denen nach dem besten Absageschreiben gesucht wird. Als ein Kuriosum galt nämlich ein richtiger Wettbewerb - der refline-Wettbewerb - den man im Jahre 2001 veranstaltet hat. Die Früchte dieses Wettbewerbs sind in einem Buch „Die besten Eis-Schreiben an Bewerber“ (2005) veröffentlicht worden, unter denen man z.B. ein solches Schreiben findet:

\section{(D) mava}

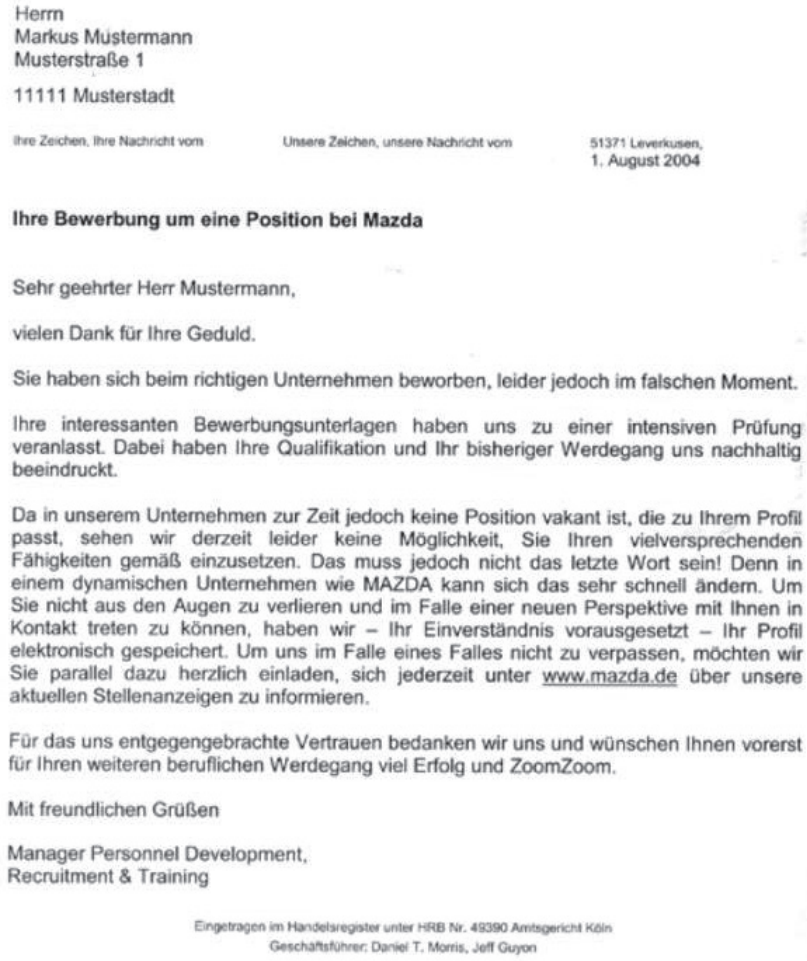

Abbildung 2. Beispiel für ein Eisschreiben

Vgl. http://www.spiegel.de/unispiegel/jobundberuf/0,1518,320552,00.html, Zugriff am 27.09.2015

${ }^{13}$ Titelfragment des Werks: Sprenzinger, Jürgen (2008): Sehr geehrter Herr Hornbach: Um ein Haar hätte ich mich bei Ihnen beworben - Absagen auf unverlangte Stellenanzeigen. München. 
Man suchte auch nach den besten Absagen, und die Gewinner wurden in der Publikation „Die besten Absageschreiben Award 2009“ veröffentlicht. Den ersten Platz nahm das Absageschreiben von Continental AG ein, und das mit folgender Begründung: „Im Inhalt sehr authentisch, in der Sprache klar und untheatralisch geht das Unternehmen der Jury zufolge in ihren Ablehnungsschreiben auf die eigene Unternehmenssituation und die Befindlichkeiten der Bewerber ein" ${ }^{\text {"14 }}$, was dem folgenden Absageschreiben zu entnehmen ist:

\footnotetext{
Continental

Sehr geehrte Frau Mustermann,

wir kommen auf thre Bewerbung zuruck und bedanken uns nochmals fur thr Interesse an einer Mitarbeit in unserem Unternehmen.

Mit Blick auf die allgemeine wirtschaftiche Situation und dem daraus resultierenden Druck fur Restrukturierungen in unserer Organisation, haben wir für diese Vakanz sehr viele interne Bewerbungen erhalten. Aufgrund der besonderen Verantwortung gegenuber unseren derzeitigen Beschaftigten haben wir uns dazu entschlossen, die Stelle intern zu besetzen.

Ihre Bewerbung ist dennoch unverandert für uns interessant, zurzeit profen wir weitere Einsatzmóglichkeiten für Sie.

Deshalb behalten wir - Ihr Einverstandnis voraussetzend - Ihre Bewerbung weiterhin in unserer Datenbank.

Eine Loschung threr Daten erfolgt, sofern wir nicht wieder mit thnen in Kontakt treten, automatisch nach einem Jahr. Sollten Sie mit der Speicherung Ihrer Daten nicht einverstanden sein, informieren Sie uns bitte umgehend.

Wir würden uns freuen, eventuell zu einem spatteren Zeitpunkt wieder mit thnen ins Gesprach zu kommen.

Vielen Dank für Ihr Verstandnis und thre Geduld.

Mit freundlichen Grusen
}
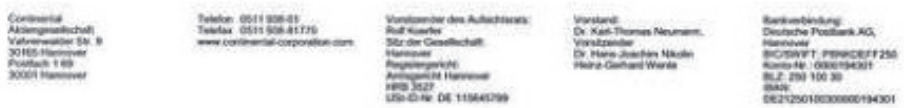

Kienbaum

Abbildung 3. Das beste Absageschreiben 2009

Vgl. http://www.hrweb.at/2012/10/niete-die-richtige-absage/, Zugriff am 27.09.2015

Ergänzend ist auch auf das 2008 im Knaur Taschenbuch Verlag veröffentlichte Buch von Jürgen Sprenzinger unter dem vielsagenden Titel: „Sehr geehrter Herr Hornbach: Um ein Haar hätte ich mich bei Ihnen beworben - Absagen auf unverlangte Stellenanzeigen" hinzuweisen. Der Autor geht auf eine witzige Weise auf ein aktuelles Thema aus dem Bereich des Personalmarketings ein, nämlich auf den Umgang mit den Absageschreiben auf Bewerbungen. Das Bewerbungsverfahren

${ }^{14}$ Vgl. http://www.hrweb.at/2012/10/niete-die-richtige-absage/, Zugriff am 27.09.2015. 
und die oft darauffolgenden Absagebriefe werden folgendermaßen kommentiert: „Bewerbungen sind ein Krampf. Wer auf Jobsuche ist, durchsucht bisweilen Hunderte Annoncen, müht sich bei den Anschreiben - und erhält zum Dank meist nur eine plumpe Absage" ${ }^{\text {"15. }}$. Diese Situation wird von Sprenzinger umkehrt, indem er Beispiele für Absagen auf Stellenanzeigen erstellt und Beispiele aus einer großen Sammlung von Absagefloskeln anführt.

\section{Schlussfolgerungen}

Wie oben gezeigt wurde, können schon im kurzen Zeitraum (2001-2012) in der Entwicklung einer Textsorte Änderungen nachgewiesen werden. In Bezug auf die Absageschreiben wurden sie vom Gesetz und vom technischen Fortschritt erzwungen.

Eines hat sich aber nicht geändert: Absage ist eine Absage und unabhängig davon, wie sie einem Bewerber übermittelt wird (Versprechen der späteren Kontaktaufnahme in den Eisschreiben, Alternativen, Vorschläge usw.), ändert es nicht an den Tatsachen. Das Greifen der Absageschreiber zu immer moderneren und einfacheren Lösungen, wie z.B. zu den Absage-Generatoren verwundert in diesem Kontext auch nicht, denn gewisse Muster und Beispiele, die in den Ratgebern, Briefstellern und Absage-Generatoren dargeboten werden, können als überprüfte Lösungen gelten. Natürlich hängt es mit einer weitgehenden Einschränkung der Eigenleistung und der Kreativität der Absageschreiber zusammen, aber es erhebt sich in diesem Zusammenhang die Frage, ob diese überhaupt erwartet werden.

Es lässt sich auch die Tendenz beobachten, dass im Laufe der Zeit die Absage immer direkter formuliert wird. Immer seltener werden dabei etwaige Begründungen, Entschuldigungen u.a. angeführt, die eigentlich nichts an den Tatsachen ändern. Das hängt mit einer gewissen Routiniertheit zusammen, die im Gegensatz $\mathrm{zu}$ dem in den Briestellern und Ratgebern geforderten Individualismus beim Behandeln von Bewerbungsunterlagen steht.

\section{LITERATURVERZEICHNIS}

Brucker M. (2008), Imagepflege für Arbeitgeber. In: Markt und Mittelstand, 01.10, Nr. 10, S. 28-39. Drescher M. (1994), Für zukünftige Bewerbungen wünschen wir Ihnen mehr Erfolg. Zur Formelhaftigkeit von Absagebriefen. In: Deutsche Sprache, H. 2, S. 117-137.

Duden (1997), Briefe gut und richtig schreiben. Ratgeber für richtiges und modernes Schreiben, Mannheim/Leipzig/Wien/Zürich.

Duden (2005a), Wzory listów niemieckich, Warszawa.

${ }^{15} \mathrm{http}: / /$ www.zeit.de/karriere/bewerbung/2010-01/absagen-an-unternehmen, Zugriff am 26.03.2015. 
Duden (2005b), Moderne Geschäftsbriefe leicht gemacht, Mannheim/Leipzig/Wien.

Ermert, K. (1979), Briefsorten. Untersuchung zu Theorie und Empirie der Textklassifikation, Tübingen.

Gansel Ch., Jürgens F. (2007), Textlinguistik und Textgrammatik. Eine Einführung, Stuttgart/Göttingen.

Grein M. (2007), Kommunikative Grammatik im Sprachvergleich. Die Sprechaktsequenz Direktiv und Ablehnung im Deutschen und Japanischen, Tübingen.

Große U. (1976), Text und Kommunikation. Eine linguistische Einführung in die Funktionen der Texte, Stuttgart.

Heinemann W., Heinemann M. (2002), Grundlagen der Textlinguistik. Interaktion - Text - Diskurs, Tübingen.

Hovermann C. (2009), Stilvolle Absagen im Geschäftsalltag: Wie Sie negative Informationen freundlichen und konstruktiv vermitteln, Bonn.

Kleb T. (2009), Die besten Absageschreiben an Bewerber, Norderstedt.

Jobware Online Service GmbH (2014), Die besten Absageschreiben an Bewerber, Norderstedt.

Lubbers B. (1982), Persönliche und geschäftliche Briefe im Privatleben. Musterbriefe und Erläuterungen für: Glückwünsche, Danksagungen, Einladungen, Zusagen, Absagen, Bewerbungen, Kündigungen, Mahnungen, Beschwerden, Behördenkorrespondenz, Beileidschreiben, Landsberg am Lech.

Markt \& Technik (2005), Gute Absageschreiben gehören zum „Employer Branding“. Gekonnt bauchpinseln. In: Markt und Technik, H. 20, S. 64.

Müller E., Moser K. (2006), Reaktionen auf Ablehnungsschreiben an Bewerber. Das Beispiel „Eisschreiben". In: Zeitschrift für Personalforschung, Bd. 20, H.4, S. 343-355.

Nixdorf N. (2002), Höflichkeit im Englischen, Deutschen, Russischen: ein interkultureller Vergleich am Beispiel von Ablehnungen und Komplimenterwiderungen, Marburg.

Redder A. (2010), Grammatik und sprachliches Handeln in der Funktionalen Grammatik. In: Grammatik und sprachliches Handeln. Japanische Gesellschaft für Germanistik, München, S. 9-24.

Refline AG und Terra Personalmarketing GmbH \& CoKG (2007), Die besten Aperitif-Briefe an Bewerber, o. O.

Sprenzinger J. (2008), Sehr geehrter Herr Hornbach: Um ein Haar hätte ich mich bei Ihnen beworben - Absagen auf unverlangte Stellenanzeigen, München.

Szczęk J. (2015), Absageschreiben auf Bewerbungen. Eine pragmalinguistische Studie, Berlin.

Szczęk J. (2015a), Auf die schönste Bewerbung kommt oft eine formelhafte Absage. Ein Beitrag zur Formelhaftigkeit der Texte am Beispiel der deutschen Absageschreiben auf Bewerbungen. W: Aussiger Beiträge 9, 2015, S. 205-228.

\section{INTERNETQUELLEN}

http://www.gesetze-im-internet.de/agg/BJNR189710006.html, Zugriff am 17.07.2012.

http://www.azubee.de/fileadmin/user_upload/.pdf/azubee_allgemein/Bewerbern_diskriminierungsfrei_absagen.pdf, Zugriff am 24.07.2013.

http://www.azubee.de/fileadmin/user_upload/.pdf/azubee_allgemein/Bewerbern_diskriminierungsfrei_absagen.pdf, Zugriff am 23.07.2013.

http://www.absage-bewerbung.de/absagen-generator-absagen-individuell-zusammenstellen/, Zugriff am 13.09.2013.

http://www.arbeitstipps.de/absage-zur-bewerbung-formulieren-muster-und-vorlagen.html\#. VgE9hZdBFnA, Zugriff am 22.09.2015. 
Von Absagebriefen zu Absagegeneratoren - Konstanz und Wandel in Form und Inhalt... 29

http://www.absage-bewerbung.de/absagen-generator-absagen-individuell-zusammenstellen/, Zugriff am 22.09.2015.

http://www.bewerbungsplan.de/absageschreiben-fuer-die-bewerbung/, Zugriff am 22.09.2015. http://www.business-solution-tools.de/business-solution-tools.de/tools/online-generatoren/ absageschreiben-bewerber/, Zugriff am 22.09.2015.

http://www.bewerbungsplan.de/absageschreiben-fuer-die-bewerbung/, Zugriff am 22.09.2015. www.spiegel.de/unispiegel/jobundberuf/html, Zugriff am 8.07.2014.

http://www.hrweb.at/2012/10/niete-die-richtige-absage/, Zugriff am 27.09.2015.

\author{
Joanna Szczęk
}

\title{
FROM REFUSAL LETTERS TO REFUSAL LETTER GENERATORS - CONSTANCY AND CHANGEABILITY IN THE FORM AND CONTENT OF GERMAN JOB APPLICATION REFUSAL LETTERS
}

(Summary)

The situation in the job market and specific expectations of job applicants lie at the heart of a peculiar "career" that has recently been experienced in refusal letters. Additional elements influencing their form and content are German law regulations. Aperitif-Briefe, Eisschreiben, and refusal letters proper are the three main variants of this text genre which has undergone noticeable evolution in the investigated period of 2001-2012. The aim of this article is to indicate the development stages of this text genre, paying special attention to the aids offered to refusal letter authors by developing technology, especially by media.

Key words: refusal letters, refusal letter generators, refusing, job applications. 